\title{
BMJ Open Rotator cuff unloading versus loading exercise program in the conservative treatment of patients with rotator cuff tear: protocol of a randomised controlled trial
}

\author{
Larissa Pechincha Ribeiro, ${ }^{1}$ Ann Cools, ${ }^{2}$ Paula Rezende Camargo (i) ${ }^{1}$
}

To cite: Ribeiro LP, Cools A, Camargo PR. Rotator cuff unloading versus loading exercise program in the conservative treatment of patients with rotator cuff tear: protocol of a randomised controlled trial. BMJ Open 2020;10:e040820. doi:10.1136/ bmjopen-2020-040820

- Prepublication history and additional materials for this paper is available online. To view these files, please visit the journal online (http://dx.doi. org/10.1136/bmjopen-2020040820).

Received 29 May 2020 Revised 05 November 2020 Accepted 20 November 2020

Check for updates

(c) Author(s) (or their employer(s)) 2020. Re-use permitted under CC BY-NC. No commercial re-use. See rights and permissions. Published by BMJ.

${ }^{1}$ Laboratory of Analysis and Intervention of the Shoulder Complex, Department of Physical Therapy, Universidade Federal de São Carlos, São Carlos, Brazil

${ }^{2}$ Department of Rehabilitation Sciences and Physical Therapy, Ghent University, Ghent, Belgium

Correspondence to Dr Paula Rezende Camargo; prcamargo@ufscar.br

\section{ABSTRACT}

Introduction Atraumatic and degenerative rotator cuff tears are common in individuals over 55 years of age. This condition can have a high impact on social life and is associated with chronic pain, weakness and dysfunction of the upper limb. There is evidence that conservative approaches should be the first treatment option. Conservative treatment usually addresses a variety of therapeutic behaviours without providing scientific arguments for the choice and progression of exercises. Objective To compare the effects of two different exercise programmes based on the load of the rotator cuff on a population with shoulder pain and rotator cuff tears. Methods and analysis This is a controlled, randomised, blinded clinical trial. Seventy-eight individuals with shoulder pain and presence of atraumatic and degenerative rotator cuff tear will participate and will be randomly distributed between two groups. The primary outcome will be quality of life (The Western Ontario Rotator Cuff Index), and secondary outcomes will include pain, function (Disabilities of the Arm, Shoulder and Hand), fear avoidance beliefs (Fear Avoidance Beliefs QuestionnaireBrazil), kinesiophobia (Tampa Scale), Pain Catastrophizing Scale, muscle strength of abductors, external and internal rotators of the shoulder, range of motion of arm elevation and patient satisfaction. The treatment will be performed for 12 weeks ( $2 x /$ week) acording to the selected group (Rotator Cuff Unloading x Rotator Cuff Loading Exercise Programme).

Ethics and dissemination The study protocol was approved by the Institutional Review Board. The findings of the trial will be disseminated through peer-reviewed journals and scientific conferences.

Trial registration number NCT03962231.

\section{INTRODUCTION}

Rotator cuff disease is one of the most common musculoskeletal disorders in the adult population. Rotator cuff tears are highly prevalent, from $22 \%$ at the age of 65 years, to more than $62 \%$ in a population over 80 years old, ${ }^{1}$ and appear to be an age-related finding on diagnostic imaging. The supraspinatus
Strengths and limitations of this study

- This study is a unique randomised controlled trial that compares rotator cuff loading versus unloading exercises on clinical aspects in patients with rotator cuff tears.

- The results of this study will likely contribute to the physical therapy's decision making on exercise prescriptions to individuals with rotator cuff tears.

- This study is randomised, prospectively registered, concelead allocation, with blinded evaluators and intention-to-treat approach.

- The results of this study cannot be extrapolated to individuals with massive rotator cuff tears.

- The results of this study cannot be extrapolated to individuals with traumatic rotator cuff tear and to those who already undergone surgical repair.

tendon is the most commonly affected tendon due to its anatomical location, tensile and compressive overload, ${ }^{2}$ vascular changes and degeneration process associated with ageing. ${ }^{3}$ Although degenerative rotator cuff tears are not necessarily symptomatic, ${ }^{4-6}$ a significant proportion of these injuries cause pain and disability of the patient over time. This condition may have a high impact in social life and can be associated to chronic pain, weakness and dysfunction of the upper extremities. ${ }^{7}$

The healthcare system usually deals with increasing expenses for rotator cuff surgery. $^{8-11}$ In addition, shoulder dysfunction is associated with high societal cost and patient burden. A recent systematic review with meta-analysis has supported that conservative approaches should be the firstline treatment for individuals with rotator cuff tears. $^{12}$ The rationale for this is that both surgical and non-surgical approaches improve function and pain in patients with tears of the rotator cuff. 
The current gap in literature consists of the lack of knowledge on which exercises are the best to perform in this population: either exercises to strengthen the remaining muscle fibres of the rotator cuff (loading the rotator cuff), or exercises focusing on strengthening the other shoulder muscles, while decreasing the tension on the rotator cuff muscles (unloading the rotator cuff). At present, both exercise types are combined in most existing treatment programs, ${ }^{13} 14$ or rotator cuff tension is not taken into consideration during exercise selection.

The aim of this study will be to compare the effects of a rotator cuff unloading exercise program compared with a rotator cuff loading exercise program on quality of life, pain, function, fear avoidance beliefs, kinesiophobia, pain catastrophising, strength, range of motion and satisfaction with treatment in patients with shoulder pain and rotator cuff tear. We believe that the unloading rotator cuff exercise program will produce better outcomes in the follow-up as regeneration of the rotator cuff is very unlikely in degenerative rotator cuff tears.

\section{METHODS AND ANALYSIS \\ Study design}

The study will be a two-arm, prospectively registered randomised controlled trial with a blinded assessor. The trial has been designed according to the Standard Protocol Items: Recommendations for Interventional Trials (SPIRIT) and Consolidated Standards of Reporting Trials (CONSORT) guidelines.

\section{Study setting}

The study will be conducted at the Laboratory of Analysis and Intervention of the Shoulder Complex located at the Department of Physical Therapy, Universidade Federal de São Carlos, São Carlos, Brazil.

\section{Eligibility criteria}

A physical therapist with 7 years of clinical experience will determine wether or not patients will be eligible to participate in the study. Individuals of both sexes, older than 55 years, with shoulder pain and atraumatic supraspinatus tendon tear documented with Magnetic Resonance Imaging or ultrasound, at least $90^{\circ}$ of active arm elevation will participate in the study.

The exclusion criteria will include individuals with history of trauma related to the onset of symptoms; history of clavicle, scapula and/or humerus fracture; pain related to the cervical spine; previous shoulder surgery; glenohumeral arthritis; inflammatory arthritis; adhesive capsulitis and cognitive disorders.

Individuals will be discontinued from the study if they present fractures, surgeries, musculoskeletal injuries or neurological diseases that prevent attendance at sessions, or get corticosteroid injection at the shoulder complex during the treatment or follow-up periods. Any additional event, as adverse effects, or use of ice and hot pack during the course of the study will be registered. Individuals who

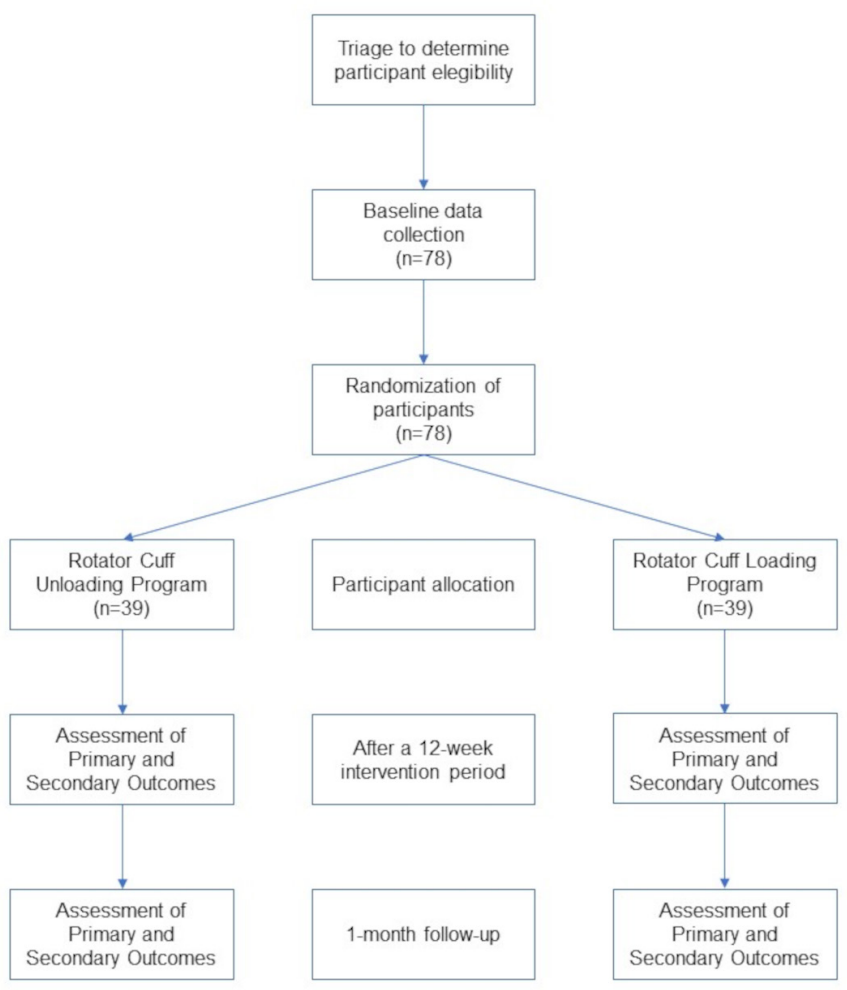

Figure 1 Study flow diagram

will be excluded, discontinued or who will complete study follow-up with remaining shoulder symptoms will receive written and verbal information about shoulder pain management and exercises.

\section{Procedure}

The participants will be recruited through flyers placed at the University, outpatient clinics and community. Advertisements in local radio, and online resources (eg, university intranet and social media) will also be used for recruitment of patients. All eligible participants will receive information about the study and will sign an informed consent form before participation. The assessor will collect the baseline data prior to randomisation, after the 12-week intervention period and 1 month after the end of treatment (follow-up). Satisfaction will only be assessed after the 12-week intervention period and at follow-up. Figure 1 brings the flow diagram of the study.

\section{Outcome measures}

The primary outcome measure will be quality of life. The secondary outcomes will be pain, function, fear avoidance beliefs, kinesiophobia, pain catastrophising, strength, range of motion and satisfaction with treatment. All scales and questionnaires have been translated and crossculturally adapted into Brazilian Portuguese.

\section{Quality of life}

The Western Ontario Rotator Cuff Index (WORC) assesses quality of life in individuals with rotator cuff disease. ${ }^{15}$ It has 21 questions in five domains: pain and physical symptoms (six questions), sports and recreation 
(four questions), work (four questions), lifestyle (four questions) and emotional state (three questions). The total score ranges from 0 to 2100 . Higher scores indicate worse quality of life. It is a reliable instrument. ${ }^{16}$

\section{Pain}

The 11-point numerical pain rating scale will be used to assess current level of shoulder pain at rest, during arm movement, and the greatest and lowest level of shoulder pain in the last week. ${ }^{17}$ The score ranges from 0 (no pain) to 10 (worst possible pain). This scale is reliable and valid for individuals with shoulder pain. ${ }^{18}$

\section{Function of the upper limbs}

The Disabilities of the Arm, Shoulder and Hand questionnaire will be used to assess upper limb function. ${ }^{19}$ It contains 30 questions that include items related to physical function, symptoms and social function. Each question has five possible answers ranging from 'no difficulty' to 'unable', and is scored on a 5-point rating scale. The maximum score is 100 , which indicates the worst possible condition. ${ }^{20}$ This questionnaire has been shown to be reliable. ${ }^{19}$

\section{Fear Avoidance Beliefs}

The Fear Avoidance Beliefs Questionnaire (FABQBrazil) ${ }^{21}$ will be used to measure fear avoidance. The FABQ-Brazil is a 6-item questionnaire that compromises two subscales physical activity and work activities. Each item is scored on a 7-point scale ranging from 'strongly agree' to 'strongly disagree'. The final scores range from 0 to 42 for physical activity subscale and from 0 to 24 for work activities subscale. ${ }^{21}$ Higher scores indicate higher beliefs in fear and avoidance. ${ }^{22}$ It has been currently used in patients with shoulder pain. ${ }^{23-25}$

\section{Kinesiophobia}

The Tampa Kinesiophobia Scale assesses the individual's fear of movement and the fear of pain recurrence. ${ }^{26} \mathrm{It}$ has 17 questions that address pain and symptom intensity. The final score ranges from 17 to 68 points. Higher scores indicate higher kinesiophobia. ${ }^{26}$ It has been used in individuals with shoulder pain and has good reliability. ${ }^{23}$

\section{Pain catastrophising}

This scale contains 13 items divided into three domains: helplessness, magnification and rumination. Total scores ranges from 0 to 52 . Higher values indicate higher degree of catastrophic thoughts. ${ }^{27}$ It has been currently used in patients with shoulder pain. ${ }^{28} 29$

\section{Muscle strength}

Strength of the shoulder abductors, ${ }^{30}$ external and internal rotators $^{31} 32$ (figure 2) will be measured using a hand-held dynamometer (Lafayette Instrument Company, Lafayette, Indiana, USA). A submaximal repetition of each test will be performed for familiarisation. Next, three $5 \mathrm{~s}$ repetitions with a 1 min rest interval between repetitions will be performed. The order of the tests will be randomised.

\section{Range of motion}

Range of motion of active arm elevation in the sagittal and scapular planes as well as the painful arcs will be assessed using a digital inclinometer ${ }^{33} 34$ (AcumarTM Lafayette Instrument Company, IN, USA). Individuals will be asked to elevate their arm in the standing position.

\section{Satisfaction}

The Global Rating of Change Scale ${ }^{35}$ will be used to assess patient's satisfaction with the treatment. It is a 15 -point numeric scale ranging from -7 (vastly worse) to 0 ('unchanged') to +7 ('vastly better'). Higher scores indicate better satisfaction. Participants will respond to the following question: 'How satisfied are you with your shoulder?' Nothing, a little, moderately or totally are the answer options to assess satisfaction.

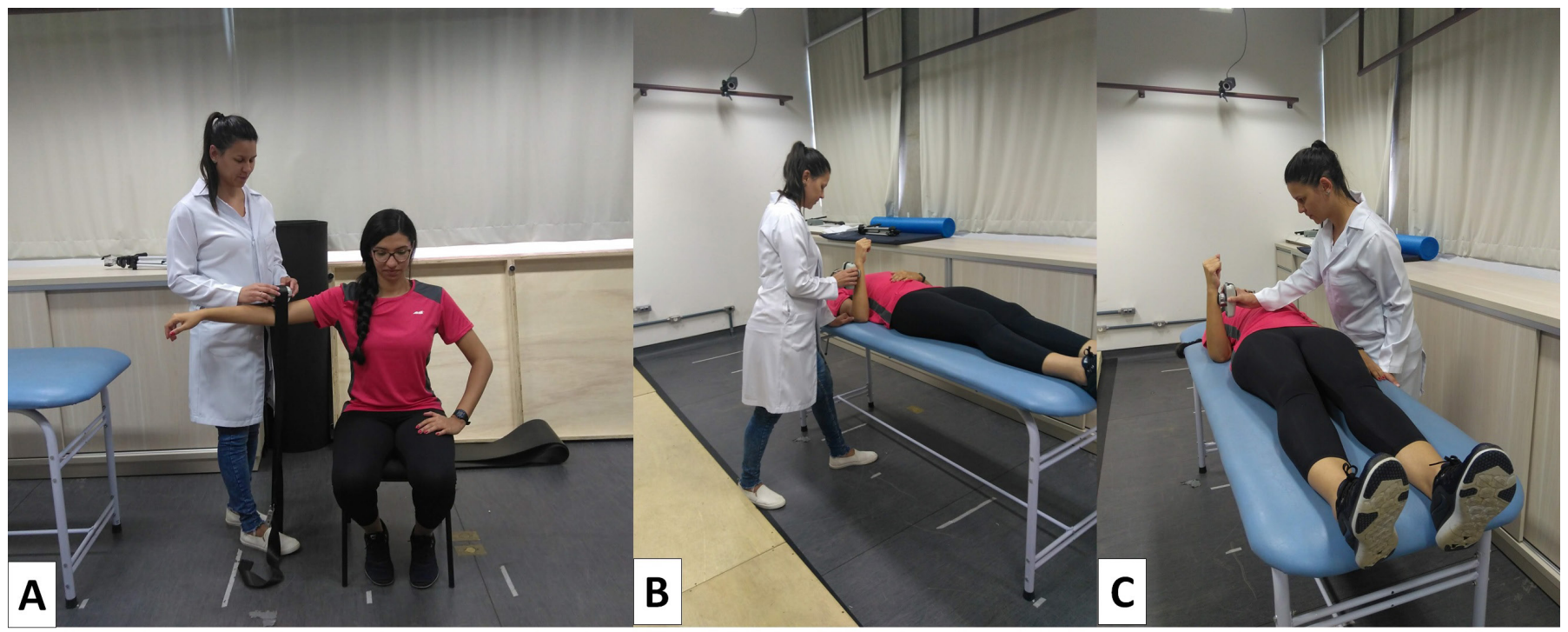

Figure 2 Muscle strength of abductors (A), external rotators (B) and internal rotators (C) of the shoulder. 


\section{Random allocation}

Patients will be randomly assigned to one of the two groups: Rotator Cuff Unloading Group or Rotator Cuff Loading Group. Randomisation will be computer based and carried out at a $1: 1$ ratio according to a random sequence generated by the website http://www.randomization.com, stratified by sex and age. An independent researcher, not involved in the treatment or assessment, will perform the randomisation process and prepare the sealed opaque envelopes with group allocation consecutively numbered. The envelopes will be securely stored and will be opened in sequence to reveal group allocation prior to the first treatment session by the researcher responsible for the treatments.

\section{Blinding}

The assessor will be blinded to treatment group assignment and the patients will be treated individually and blinded to the study hypothesis.

\section{Interventions}

Each intervention program will consist of 24 supervised sessions, which will be executed two times per week for 12 weeks. Both groups will receive advice with respect to pain control, posture and range of motion. Adherence to treatment and assessment sessions will be encouraged at each session.

The exercise programme for both groups will be based on a systematic review, ${ }^{36}$ providing a continuum of exercises, from low rotator cuff to high rotator cuff load. The selected exercises for the Rotator Cuff Unloading Group will be based on a muscle activation equal to or less than $20 \%$ of the supraspinatus maximal activity. ${ }^{36}$ The selected exercises for the Rotator Cuff Loading Group will be based on a muscle activation equal to or greater than $40 \%$ of the supraspinatus maximal activity. All exercises will be performed in 3 sets of 10 repetitions with a 1 min interval between repetitions.

\section{Rotator cuff unloading exercise program}

Patients in this group will perform four exercises: semiclosed kinetic chain elevation, deltoid reeducation, ${ }^{37}$ assisted arm elevation and an exercise for scapular control. $^{38} 39$ The exercises and their progressions are described in online supplemental appendix.

\section{Rotator cuff loading exercise program}

Patients in this group will perform conventional exercises focusing on external rotation, internal rotation and arm elevation. The exercises and their progressions are described in online supplemental appendix.

\section{Participant timeline}

The flow chart summarising procedures and patients is shown in figure 1 .

\section{Sample size}

The sample size calculation was based on the smallest significant difference of 282.6 points from the WORC ${ }^{40}$ with an standard deviation of 400 points, a power of $80 \%$, and a significance level of $5 \%$. WORC was selected because it evaluates the quality of life of individuals with rotator cuff disease. Accounting for a 15\% drop-out, 78 subjects will be included in the study, randomly allocated to two treatment groups: Rotator Cuff Unloading Exercise Programme $(n=39)$ and Rotator Cuff Loading Exercise Programme $(n=39)$.

\section{Data management, monitoring and sharing}

All data from recruitment, characteristics of the individuals who will complete or drop-out the study will be entered into an electronic form by the researchers, and the integrity and validity of the data will be verified. Identification of possible patients will be done only by the researchers. The research team is trained to address the eligibility criteria during the contact about survey made by email, phone calls and messages. Also, the team is trained about how and when to contact them for follow-up and data collection.

The results of this article will be shared (text, tables, figures, appendices) immediately after publication. No interim analyses have been planned. To request data you will need to sign a data access agreement and the request can be made by email. The changes made to the protocol will be communicated to ethics committee and also be included in the clinical trial register.

\section{Patient and public involvement}

The patients were not involved in the design, will not be involved in the conduction and dissemination of the research. The results will be sent by email or telephone in an unscientific language so that all patients understand the study's conclusions and know how to maintain self-care.

\section{Statistical analysis}

For data analysis, the statistical program Statistical Package for the Social version 23 will be used. Continuous data will be presented as mean, SD and mean difference between groups with $95 \%$ confidence interval, and categorical by frequency and percentage. Data normality will be verified by Kolmogorov Smirnov test and observation of histograms for each variable in each group. Statistical analysis will follow the principles of intention-to-treat analysis. Linear mixed models will be used to calculate differences between groups using the terms of group interaction (Rotator Cuff Unloading Group vs Rotator Cuff Loading Group) vs time (pre-treatment, 12 weeks at the end of treatment and a month after the end of treatment) for each variable.

\section{ETHICS AND DISSEMINATION Ethical aspects}

This study was approved by the Human research Ethics Committee of the University (CAAE: 
12899719.5.0000.5504) and prospectively registered at clinicaltrials.gov (NCT03962231) on September 2019.

\section{Dissemination}

The study will be disseminated through publication in peer-reviewed international journals, as well as presentations at national and international conferences.

\section{DISCUSSION}

\section{Potential impact and significance of the study}

Degenerative tears in the rotator cuff is a common finding in imaging studies due to the natural ageing process. ${ }^{41}$ There is evidence that therapeutic exercises should be the first treatment option for these patients ${ }^{14}$ because the surgical approach is not clinically superior than the non-surgical approach in this population. ${ }^{12}$ Several exercise protocols have been proposed for this population. ${ }^{14}$ However, there is no consensus on which exercises or exercise programs are the most effective. This fact challenges the physical therapist in the clinical decision making. Thus, the results of this study will likely contribute to the physical therapy's decision making on exercise prescriptions to individuals with rotator cuff tears.

\section{Strengths and weaknesses of the study}

The strength of the current study is that it is a randomised controlled trial that has been prospectively registered. Furthermore, the study includes concealed allocation and an intention-to-treat approach. The sample size has been calculated to provide appropriate statistical power to detect differences between the two treatment programs.

The assessor responsible for collecting outcome data will be blinded to treatment group assignment. Physical therapists responsible for treatment have similar clinical experience and have been trained by the main author of the study. However, the study has some limitations. Participants and therapists cannot be blinded. Both exercise programs include home guidelines, which depend on each participant's motivation. It is not possible to predict the amount of home guidelines and cryotherapy that will be performed by each group.

\section{Contribution to the physical therapy profession and to patients}

The results of this study will provide scientific basis to support physical therapists in the treatment of individuals with rotator cuff tears, helping in the choice of exercises and their progression. In addition, the findings may also help healthcare providers and patients with rotator cuff tears to reduce health costs, avoiding the need for surgery and the use of analgesic drugs. Participants will be taught how to modify their daily activities by modifying the movements and postures that appear to increase shoulder symptoms. The participants also receive a series of exercises to be performed at home in the follow-up. It is expected that patients will become independent and more empowered with good quality of life and function of the upper limbs.

Finally, the findings of the current study may contribute to a better understanding of the efficacy of exercise program for individuals with rotator cuff tears.

Contributors All authors (LPR, AC and PRC) were equally responsible for outlining the conception and protocol design. LPR, AC and PRC drafted the work, revised it critically for important intellectual content, and approved the final version.

Funding Coordenação de Aperfeiçoamento de Pessoal de Nível Superior and Conselho Nacional de Desenvolvimento Científico e Tecnológico (144436/2019-1).

Competing interests None declared.

Patient consent for publication Obtained.

Provenance and peer review Not commissioned; externally peer reviewed.

Supplemental material This content has been supplied by the author(s). It has not been vetted by BMJ Publishing Group Limited (BMJ) and may not have been peer-reviewed. Any opinions or recommendations discussed are solely those of the author(s) and are not endorsed by BMJ. BMJ disclaims all liability and responsibility arising from any reliance placed on the content. Where the content includes any translated material, BMJ does not warrant the accuracy and reliability of the translations (including but not limited to local regulations, clinical guidelines, terminology, drug names and drug dosages), and is not responsible for any error and/or omissions arising from translation and adaptation or otherwise.

Open access This is an open access article distributed in accordance with the Creative Commons Attribution Non Commercial (CC BY-NC 4.0) license, which permits others to distribute, remix, adapt, build upon this work non-commercially, and license their derivative works on different terms, provided the original work is properly cited, appropriate credit is given, any changes made indicated, and the use is non-commercial. See: http://creativecommons.org/licenses/by-nc/4.0/.

ORCID iD

Paula Rezende Camargo http://orcid.org/0000-0002-8961-4353

\section{REFERENCES}

1 Teunis T, Lubberts B, Reilly BT, et al. A systematic review and pooled analysis of the prevalence of rotator cuff disease with increasing age. J Shoulder Elbow Surg 2014;23:1913-21.

2 Bunker T. Rotator cuff disease. Curr Orthop 2002;16:223-33.

3 Spargoli G. Supraspinatus tendon pathomechanics: a current concepts review. Int J Sports Phys Ther 2018;13:1083-94.

4 Minagawa $\mathrm{H}$, Yamamoto $\mathrm{N}$, Abe $\mathrm{H}$, et al. Prevalence of symptomatic and asymptomatic rotator cuff tears in the general population: from mass-screening in one village. J Orthop 2013;10:8-12.

5 Moosmayer S, Tariq R, Stiris MG, et al. MRI of symptomatic and asymptomatic full-thickness rotator cuff tears. A comparison of findings in 100 subjects. Acta Orthop 2010;81:361-6.

6 Barreto RPG, Braman JP, Ludewig PM, et al. Bilateral magnetic resonance imaging findings in individuals with unilateral shoulder pain. J Shoulder Elbow Surg 2019;28:1699-706.

7 Lowe CJM, Moser J, Barker K. Living with a symptomatic rotator cuff tear "bad days, bad nights": A qualitative study. BMC Musculoskelet Disord 2014;15:1-10.

8 Malavolta EA, Assunção JH, Beraldo RA. Pinto G de MR, Gracitelli MEC, Ferreira Neto AA. Rotator cuff repair in the Brazilian unified health system: Brazilian trends from 2003 to 2015. Rev Bras Ortop (English Ed 2017;52:501-5.

9 Syed UAM, Aleem AW, Wowkanech C, et al. Neer Award 2018: the effect of preoperative education on opioid consumption in patients undergoing arthroscopic rotator cuff repair: a prospective, randomized clinical trial. J Shoulder Elb Surg 2018;27:962-7.

10 Westermann RW, Anthony CA, Bedard N, et al. Opioid consumption after rotator cuff repair. Arthrosc - J Arthrosc Relat Surg 2017;33:1467-72.

11 Uquillas CA, Capogna BM, Rossy WH, et al. Postoperative pain control after arthroscopic rotator cuff repair. J Shoulder Elb Elbow Surgery 2016;25:1204-13.

12 Piper CC, Hughes AJ, Ma Y, et al. Operative versus nonoperative treatment for the management of full-thickness rotator cuff tears: a systematic review and meta-analysis. J Shoulder Elbow Surg 2018;27:572-6. 
13 Kuhn JE, Dunn WR, Sanders R, An Q, et al. Effectiveness of physical therapy in treating atraumatic full-thickness rotator cuff tears: a multicenter prospective cohort study. J Shoulder Elbow Surg 2013;22:1371-9.

14 Jeanfavre M, Husted S, Leff G. Exercise therapy in the non-operative treatment of full-thickness rotator cuff tears: a systematic review. Int J Sports Phys Ther 2018;13:335-78.

15 Lopes A, Standniki S, Masiero D, et al. Tradução E Adaptação Cultural Do Worc : Um Questionário De Qualidade De Vida Para Alterações Do Manguito Rotador. Rev bras fisioter 2006;3:309-15.

16 Lopes AD, Ciconelli RM, Carrera EF, et al. Validity and reliability of the Western Ontario rotator cuff index (WORC) for use in Brazil. Clin $J$ Sport Med 2008;18:266-72.

17 Jensen MP, Turner JA, Romano JM, et al. Comparative reliability and validity of chronic pain intensity measures. Pain 1999;83:157-62.

18 Mintken PE, Glynn P, Cleland JA. Psychometric properties of the shortened disabilities of the arm, shoulder, and hand questionnaire (QuickDASH) and numeric pain rating scale in patients with shoulder pain. J Shoulder Elbow Surg 2009;18:920-6.

19 Orfale AG, Araújo PMP, Ferraz MB, et al. Translation into Brazilian Portuguese, cultural adaptation and evaluation of the reliability of the disabilities of the arm, shoulder and hand questionnaire. Braz J Med Biol Res 2005;38:293-302.

20 Hudak PL, Amadio PC, Bombardier C. Development of an upper extremity outcome measure: the DASH (disabilities of the arm, shoulder, and head). Am J Ind Med 2017;608:527-46.

21 Abreu AM de, Faria CDC de M, Cardoso SMV, et al. Versão brasileira do fear avoidance beliefs questionnaire. Cad. Saúde Pública 2008:24:615-23.

22 de Souza FS, Marinho CdaS, Siqueira FB, et al. Psychometric testing confirms that the Brazilian-Portuguese adaptations, the original versions of the Fear-Avoidance beliefs questionnaire, and the Tampa scale of Kinesiophobia have similar measurement properties. Spine 2008;33:1028-33.

23 Mintken PE, Cleland JA, Whitman JM, et al. Psychometric properties of the Fear-Avoidance beliefs questionnaire and Tampa scale of Kinesiophobia in patients with shoulder pain. Arch Phys Med Rehabil 2010;91:1128-36.

24 Riley SP, Tafuto V, Cote M, et al. Reliability and relationship of the fear-avoidance beliefs questionnaire with the shoulder pain and disability index and numeric pain rating scale in patients with shoulder pain. Physiother Theory Pract 2019;35:1-7.

25 Woollard JD, Bost JE, Piva SR, et al. The ability of preoperative factors to predict patient-reported disability following surgery for rotator cuff pathology. Disabil Rehabil 2017;39:2087-96.

26 Siqueira FB, Teixeira-Salmela LF, Magalhães LDC. Análise das propriedades psicométricas dA versão brasileira dA escala Tampa de cinesiofobia. Acta ortop. bras. 2007:15:19-24.

27 Sehn F, Chachamovich E, Vidor LP, et al. Cross-Cultural adaptation and validation of the Brazilian Portuguese version of the pain catastrophizing scale. Pain Med 2012;13:1425-35.
28 George SZ, Hirsh AT. Psychologic influence on experimental pain sensitivity and clinical pain intensity for patients with shoulder pain. $J$ Pain 2009;10:293-9.

29 George SZ, Staud R, Borsa PA, et al. Biopsychosocial influence on shoulder pain: rationale and protocol for a pre-clinical trial. Contemp Clin Trials 2017;56:9-17.

30 Katoh M. Test-retest reliability of isometric shoulder muscle strength measurement with a handheld dynamometer and belt. J Phys Ther Sci 2015;27:1719-22.

31 Schrama PPM, Stenneberg MS, Lucas C, et al. Intraexaminer reliability of hand-held dynamometry in the upper extremity: a systematic review. Arch Phys Med Rehabil 2014;95:2444-69.

32 Cools AMJ, Vanderstukken F, Vereecken F, et al. Eccentric and isometric shoulder rotator cuff strength testing using a hand-held dynamometer: reference values for overhead athletes. Knee Surg Sports Traumatol Arthrosc 2016;24:3838-47.

33 Kolber MJ, Hanney WJ. The reliability and concurrent validity of shoulder mobility measurements using a digital inclinometer and goniometer: a technical report. Int J Sports Phys Ther 2012;7:306-13.

34 Kolber MJ, Vega F, Widmayer K, et al. The reliability and minimal detectable change of shoulder mobility measurements using a digital inclinometer. Physiother Theory Pract 2011;27:176-84.

35 Kamper SJ, Maher CG, Mackay G. Global rating of change scales: a review of strengths and weaknesses and considerations for design. Journal of Manual \& Manipulative Therapy 2009;17:163-70.

36 Edwards PK, Ebert JR, Littlewood C, et al. A systematic review of electromyography studies in normal shoulders to inform postoperative rehabilitation following rotator cuff repair. $J$ Orthop Sports Phys Ther 2017;47:931-44.

37 Levy O, Mullett $\mathrm{H}$, Roberts S, et al. The role of anterior deltoid reeducation in patients with massive irreparable degenerative rotator cuff tears. J Shoulder Elbow Surg 2008;17:863-70.

38 Kibler WB, Sciascia AD, Uhl TL, et al. Electromyographic analysis of specific exercises for scapular control in early phases of shoulder rehabilitation. Am J Sports Med 2008;36:1789-98.

39 De Mey K, Danneels L, Cagnie B, et al. Kinetic chain influences on upper and lower trapezius muscle activation during eight variations of a scapular retraction exercise in overhead athletes. J Sci Med Sport 2013;16:65-70.

40 Gagnier JJ, Robbins C, Bedi A, et al. Establishing minimally important differences for the American shoulder and elbow surgeons score and the Western Ontario Rotator Cuff Index in patients with full-thickness rotator cuff tears. J Shoulder Elbow Surg 2018;27:e160-6.

41 Fehringer EV, Sun J, VanOeveren LS, et al. Full-Thickness rotator cuff tear prevalence and correlation with function and co-morbidities in patients sixty-five years and older. J Shoulder Elbow Surg $2008 ; 17: 881-5$ 